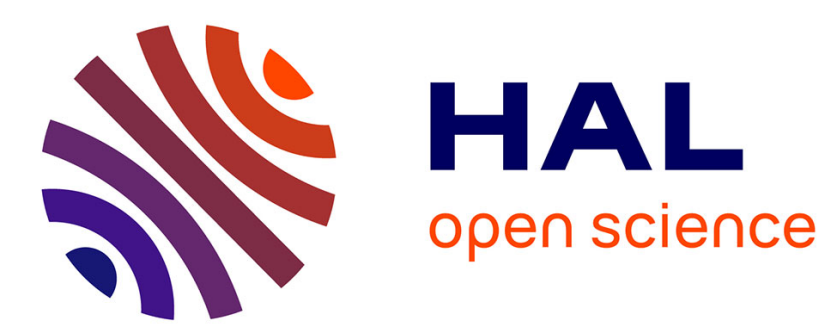

\title{
Effect of Loading Induced Anisotropy on the Shear Behavior of Rough Interfaces
}

\author{
Anil Misra, Shiping Huang
}

\section{To cite this version:}

Anil Misra, Shiping Huang. Effect of Loading Induced Anisotropy on the Shear Behavior of Rough Interfaces. 2011. hal-00555552

\section{HAL Id: hal-00555552 \\ https://hal.science/hal-00555552}

Preprint submitted on 13 Jan 2011

HAL is a multi-disciplinary open access archive for the deposit and dissemination of scientific research documents, whether they are published or not. The documents may come from teaching and research institutions in France or abroad, or from public or private research centers.
L'archive ouverte pluridisciplinaire HAL, est destinée au dépôt et à la diffusion de documents scientifiques de niveau recherche, publiés ou non, émanant des établissements d'enseignement et de recherche français ou étrangers, des laboratoires publics ou privés. 


\section{Effect of Loading Induced Anisotropy on the Shear Behavior of \\ Rough Interfaces}

Anil Misra and Shiping Huang

Department of Civil, Environmental and Architectural Engineering, the University of

Kansas

\section{Corresponding Author:}

Dr. Anil Misra

Professor, Civil, Environmental and Architectural Engineering Department

The University of Kansas

Learned Hall

1530 W. 15th Street

Lawrence, KS 66045-7609

Ph: (785) 864-1750

Fax: (785) 864-5631

Email: amisra@ku.edu 


\title{
Effect of Loading Induced Anisotropy on the Shear Behavior of Rough Interfaces
}

\author{
Anil Misra and Shiping Huang \\ Department of Civil, Environmental and Architectural Engineering, the University of \\ Kansas
}

\begin{abstract}
We have utilized a statistical method to model the shear behavior of rough contacts. In contrast to the traditional statistical methods, which only describe the asperity height and curvature distributions, we have introduced a contact orientation distribution in our analysis. We have also incorporated asperity contact sliding and developed an incremental scheme for computing the stress-displacement relationship. Using this enhanced model, we can demarcate the boundary between elastically deforming and sliding asperity contact orientations under given loading. Consequently, we can describe the shear-normal coupling as well as the effects of inherent anisotropy and the induced anisotropy of the sheared interfaces.
\end{abstract}

Keywords: contact mechanics; sliding; elastic; roughness 


\section{Introduction}

Stress-displacement behavior of contact between solid bodies has been one of the most widely researched problems with contributions spanning more than a century[1-4]. It has been widely recognized that surface roughness has a significant role in determining the contact behavior under loading that is in the direction normal and tangential to the nominal contact surface. A clear consequence of surface roughness is that the actual contact area at the interface is smaller than the area of contacting surfaces because the contact occurs via asperities. Moreover, when the surfaces come into contact, the asperity heights and their relative locations are uncertain, consequently, the resulting asperity contacts are oblique to the nominal contact directions and of random orientations. A large number of models have been proposed to simulate the interface behavior of contacting solids. The recent approaches of rough contact modeling can be considered in three categories based upon how the surface roughness is incorporated in the calculations: (1) direct simulation using the finite element method, (2) fractal representation, and (3) statistical methods.

Direct simulation of rough contact is useful for providing perceptual intuition and showing details of the local behavior at the interfaces [4-9]. Furthermore, it is able to consider the elasto-plastic behavior of material as well as larger deformation. However, direct simulation can entail prohibitive computational expense for modeling surfaces with numerous asperities of irregular geometry. Consequently, this approach is seldom used to model the whole contact surface, although it could be useful in micro-macro approaches for determining the relationships between local and global properties. Along the lines of fractal representation, a number of authors have shown that the rough interface demonstrates the self-affine feature which indicates the geometry of the interface are scale dependent [7, 10-13]. The fractal model was proposed to account for such feature. However, the fractal model typically does not incorporate the local asperity behavior which is important when considering the shear 
behavior. Furthermore, many materials like thin-film disks, magnetic tapes and geotechnical materials have been shown to have little scale dependency and can be adequately described by the statistical approach [14]. In contrast to the fractal approach, the statistical approach is an asperity based model, in which the interface behavior is described as a group effect of numerous local asperity contacts [15-31]. Scale dependent parameters have also been considered within the ambit of statistical approaches to address the fractal or resolution dependent nature of rough surfaces [32, 33]. The pioneering work on the statistical approach was done by Greenwood and Williams[15], who assumed that the surface is formed by a large number of spherical shaped asperities with equivalent radius but different heights that follow Gaussian distribution. Nayak [18, 34] introduced the techniques of random process theory into the analysis of Gaussian roughness. In order to account for anisotropic interface, McCool and Gassel [22] introduced the elliptic paraboloid asperities instead of spherical shape asperities. However, the basic solution for elliptic paraboloid asperities is much more complicated than that for spherical asperities, which has been found to adequately describe the asperity contact behavior. The advantage of the statistical method is that it not only considers the local behavior of contacting surface, but also describes the interface geometry in a simple way. Therefore, this method continues to be attractive for describing rough surface contact behavior.

In this paper, we introduce an orientation function to consider the anisotropic surface while retaining the spherical shape of the asperities in the context of the statistical approach. The orientation function models the random asperity contact obliquity which results in the coupling of the shear and normal directions which is commonly ignored in other models. Furthermore, we incorporate Hertz-Mindlin fundamental solutions and sliding at the asperity contact in our model. This work extends the previous micromechanical approach developed by the authors $[28,35,36]$. A numerical procedure is implemented to evaluate the derived expressions for the overall contact behavior. We then obtain the contact behavior under normal-shear combined loading. In particular, we investigate (1) how the stress-displacement 
relationship evolves as the interface is subjected to shear loading whose direction is varied, and (2) the coupling between the normal and shear behavior under shear loading.

In the subsequent discussion, we first briefly present our approach for modeling contact behavior, including the statistical modeling of contact surface and the essence of the micromechanical approach. We then utilize the micromechanical model to investigate the sliding behavior of asperity contacts and the overall stress-displacement behavior of the interface. We find that under certain applied loading, existing asperity contacts can unload in the normal direction and separate. Similarly, contacts that are sliding can unload in the shear direction and such that they are no longer sliding. We also find that the overall behavior of the interface exhibits significant coupling between the normal-shear and shear-shear directions depending upon the loading sequence.

\section{Modeling Method}

\subsection{Statistical Description of Contact Interface}

The contact surface geometry determines the orientations and the number of asperity contacts under a given loading condition. The composite topography of contacting surfaces, described via statistics of asperity contact heights, orientations, and curvatures may be utilized for this purpose $[9,18,21,28]$. In this paper, the statistics of asperity contact heights is described via gamma distributions, asperity contact orientations via spherical harmonic expansions, and asperity curvatures are assumed to be constant for simplicity. It is usual to define the asperity contact height with reference to the highest peak of the composite topography such that, asperity contact height, $r$, represents the overlap of the interacting surfaces. The density function for asperity contact heights, $H(r)$, is given by a gamma distribution [21, 26, 27] expressed as: 


$$
H(r)=\frac{r^{\alpha} e^{-r / \beta}}{\Gamma(\alpha+1) \beta^{\alpha}} \quad(0<r<\infty, \alpha, \beta>0)
$$

where $\alpha$ and $\beta$ are parameters related to the mean and variance of the asperity contact heights as follows

$$
\begin{array}{ll}
\text { mean }: & r_{m}=(\alpha+1) \beta \\
\text { variance }: & r_{\sigma}^{2}=(\alpha+1) \beta^{2}
\end{array}
$$

Parameter $\alpha$ is unit less while parameter $\beta$ takes the unit of asperity contact height. Surfaces that have smaller average asperity contact height and narrow distributions of asperity contact heights are considered to be smoother. Fig. 1 gives examples of asperity contact height distributions for two interfaces that can be described as smooth and rough in comparison with each other. For an interface with $N$ asperity contacts per unit area, $N H(r) d r$ denotes that number of asperity contacts in the interval represented by $r$ and $r+d r$. Thus, the total number of asperity contacts, under a given closure, is given by

$$
N_{r}=\int_{0}^{r} N H(r) d r
$$

In order to describe the orientation distribution, we introduce a local Cartesian coordinate system as shown in Fig. 2. The local coordinate system consists of three vectors $\boldsymbol{n}, \boldsymbol{s}$ and $\boldsymbol{t}$, among which $\boldsymbol{n}$ is the vector normal to the asperity contact surface, and $s$ and $t$ are on the plane tangential to the asperity contact surface. The relationship between the local coordinate system and the global Cartesian system is given by:

$$
\begin{aligned}
& n=\langle\cos \theta, \quad \sin \theta \cos \phi, \quad \sin \theta \sin \phi\rangle \\
& s=\langle-\sin \theta, \quad \cos \theta \cos \phi, \quad \cos \theta \sin \phi\rangle \\
& t=\langle 0, \quad-\sin \theta, \quad \cos \phi\rangle
\end{aligned}
$$

The asperity contact orientation is defined by considering the inclination of the asperity contact normal with respect to that of the interface normal direction. As shown in Fig. 2, the orientation of an oblique asperity contact is defined by the azimuthal angle, $\phi$, and the meridional angle, $\theta$, measured with respect to a Cartesian 
coordinate system in which direction 1 is normal to the interface. A 3-dimensional density function utilizing shifted spherical harmonics expansion in spherical polar coordinates that describes the concentrations of asperity contact orientations was introduced by Misra [28, 35]. For an interface with isotropic distribution of asperity contact orientations, the density function, $\xi(\theta, \phi)$ is given by

$$
\begin{aligned}
& \xi(\theta, \phi)=\frac{a \sin a \theta}{2 \pi \sin \theta}\left[1+\frac{b}{4}(3 \cos a \theta+1)+3 c(\sin a \theta)^{2} \cos 2 \phi\right] \\
& \left(0 \leq \theta \leq \frac{\pi}{2 a} ; \quad 0 \leq \phi \leq 2 \pi ; \quad a \geq 1\right)
\end{aligned}
$$

where angles $\phi$ and $\theta$ are defined in Fig 2, and parameters $a, b$ and $c$ determine the shape of the density function $\xi(\theta, \phi)$. Further, to ensure that the density function is positive semi-definite, i.e. $\xi(\theta, \phi) \geq 0$, the values of parameters $b$ and $c$ are bounded as follows: $-1 \leq b \leq 2$ and $-\frac{1}{3}+\frac{b}{6} \leq c \leq \frac{1}{3}-\frac{b}{6}$. Thus, the product $N_{r} \xi(\theta, \phi) \sin \theta d \theta d \phi$ denotes the number of asperity contacts $N_{\Omega}$ in the interval represented by $\sin \theta d \theta d \phi$, that is

$$
N_{\Omega}=N_{r} \xi(\theta, \phi) \sin \theta d \theta d \phi
$$

The density function in Eq.(6) has the ability to model surfaces with varying roughness. As discussed in Misra [35] for smooth surfaces the asperity contact directions have a greater tendency to be aligned in the direction normal to the interface in contrast to that for rough surfaces. It is noteworthy that, as parameter, $a$, increases, the contact distribution concentrates towards the direction normal to the interface. In particular, the density function, $\xi(\theta, \phi)$, behaves like a delta function in the limit $a \rightarrow \infty$ and yields an expectation $E[\theta]=0$, which represents a concentrated contact orientation, normal to a perfectly smooth interface. The parameter, $a$, describes the extent of the asperity contacts in the meridional direction as well as the mean asperity contact orientation. The extent of asperity contact inclination in meridional direction is $\pi / 2$ for $a=1$ and $\pi / 4$ for $a=2$. The shapes of contact distributions vary with the values of parameter $b$, within the meridional extent of asperity contacts. The lower bound of parameter $b=-1$, represents an interface on 
which the asperity contacts tend to orient closer to the horizon. On the other hand, the upper bound of parameter $b=2$, represents an interface on which preferred orientation is closer to the interface normal. The asperity contacts are equally distributed in the meridional direction for parameter $b=0$. Parameter, $c$, describes the shape of the contact distributions in the azimuthal direction and, therefore models the inherent anisotropy in the interface tangential direction. Fig. 3 shows the 3-dimensional distribution density under different combination of parameters $a, b$ and $c$.

\subsection{Micromechanical Stress-Displacement Relationship}

In the kinematically driven approach, we assume that the asperity contact displacement, $\delta_{j}$, at a given asperity contact height is the same and directly related to the overall displacement of the interface, $\Delta_{j}$. The subscripts in this paper follow the established tensor convention unless specified otherwise. Thus, the asperity contact displacement in the local coordinate system can be written in terms of the overall interface displacements as follows:

$\left\{\begin{array}{l}\delta_{n} \\ \delta_{s} \\ \delta_{t}\end{array}\right\}=\left[\begin{array}{lll}n_{1} & n_{2} & n_{3} \\ s_{1} & s_{2} & s_{3} \\ t_{1} & t_{2} & t_{3}\end{array}\right]\left\{\begin{array}{c}\Delta_{1}-r \\ \Delta_{2} \\ \Delta_{3}\end{array}\right\}$

Note that we assume the asperities to have spherical shape with the same radius but different heights. Therefore, for a normal interface displacement $\Delta_{l}$, the displacement of the asperity contact at height $r$ is $\Delta_{l}-r$ as depicted in Fig. 4. In local coordinate system, considering the Hertz-Mindlin contact theory of perfectly smooth elastic interfaces as well as other theories of smooth elastic-plastic interfaces[3], it is reasonable to assume that normal asperity contact stiffness $K_{n}$ depends on the normal asperity contact displacement $\delta_{n}$ according to the following power law:

$K_{n}=\lambda K \delta_{n}^{\eta}$

Where $K, \lambda$ and $\eta$ are constants. The asperity contact stiffness, $K_{n}$ given by Eq.(8),becomes identical with the Hertz stiffness for contact of perfectly smooth elastic spheres when[3] 


$$
\lambda=\frac{2-v}{2(1-v)} ; \quad \eta=\frac{1}{2} ; \quad K=\frac{8 G \sqrt{R}}{3(2-v)}
$$

where $G$ is the shear modulus, $v$ is Poisson's ratio and $R$ is asperity radius of curvature. It is noteworthy that the exponent $\eta$ can vary from 0 for perfectly plastic to $1 / 2$ for perfectly elastic behavior at contact of perfectly smooth spherical asperities[3]. Since this paper focuses on monotonic loading of interfaces, we consider the case of constant normal asperity contact force and monotonically increasing asperity contact shear force. Mindlin and Deresiewicz [37] have derived the following asperity contact force-displacement relationship for this loading condition, considering partial slip at contact edge with increasing contact shear displacement:

$f_{s t}=\mu K_{n} \delta_{n}\left[1-\left(1-\frac{\delta_{s t}}{\lambda \mu \delta_{n}}\right)^{\frac{3}{2}}\right]$

Where $f_{s t}$ is the asperity contact shear force and $\delta_{s t}$ is the asperity contact shear displacement given by,

$\delta_{s t}=\sqrt{\delta_{s}^{2}+\delta_{t}^{2}}$

Thus, in s- and t-direction, we have the following force displacement relationship:

$$
\begin{aligned}
& f_{s}=\mu K_{n} \delta_{n}\left[1-\left(1-\frac{\delta_{s t}}{\lambda \mu \delta_{n}}\right)^{\frac{3}{2}}\right] \frac{\delta_{s}}{\delta_{s t}}=K_{s t}^{e} \delta_{s} \\
& f_{t}=\mu K_{n} \delta_{n}\left[1-\left(1-\frac{\delta_{s t}}{\lambda \mu \delta_{n}}\right)^{\frac{3}{2}}\right] \frac{\delta_{t}}{\delta_{s t}}=K_{s t}^{e} \delta_{t}
\end{aligned}
$$

where $K_{s t}$ is the stiffness in the tangential direction, and the superscript $e$ denotes asperity contacts that are not sliding. We note Eqs. (12) and (13) are valid when $\left|\lambda \mu \delta_{n}\right|>\delta_{s t}$. When this condition is violated, sliding occurs at the contact per the Amonton-Coulomb's friction law. In this case Eqs. (12) and (13) can be rewritten as:

$$
\begin{aligned}
& f_{s}=\mu K_{n} \delta_{n} \frac{\delta_{s}}{\delta_{s t}}=K_{s t}^{p} \delta_{s} \\
& f_{t}=\mu K_{n} \delta_{n} \frac{\delta_{t}}{\delta_{s t}}=K_{s t}^{p} \delta_{t}
\end{aligned}
$$


where the superscript $p$ denotes asperity contacts that are sliding. In Eqs. (12) through (15) the ratios $\delta_{s} / \delta_{s t}$ and $\delta_{t} / \delta_{s t}$ give the projection of the asperity contact shear force, $f_{s t}$, in the s- and the t-directions and the stiffness, $K_{s t}$, has been introduced for convenience. Thus, for a single asperity contact the force-displacement can be written in the following matrix form in the local coordinate system, where the superscript has been dropped:

$$
\left\{\begin{array}{l}
f_{n} \\
f_{s} \\
f_{t}
\end{array}\right\}=\left[\begin{array}{ccc}
K_{n} & 0 & 0 \\
0 & K_{s t} & 0 \\
0 & 0 & K_{s t}
\end{array}\right]\left\{\begin{array}{l}
\delta_{n} \\
\delta_{s} \\
\delta_{t}
\end{array}\right\}
$$

Or in the global coordinate system, the asperity contact forces, $f_{i}$, and displacements, $\delta_{j}$, are related as follows:

$$
\left\{\begin{array}{l}
f_{1} \\
f_{2} \\
f_{3}
\end{array}\right\}=\left[\begin{array}{lll}
K_{11} & K_{12} & K_{13} \\
K_{12} & K_{22} & K_{23} \\
K_{13} & K_{23} & K_{33}
\end{array}\right]\left\{\begin{array}{c}
\Delta_{1}-r \\
\Delta_{2} \\
\Delta_{3}
\end{array}\right\}
$$

Where the asperity contact stiffnesses, $K_{i j}$, given by:

$$
K_{i j}=K_{n} n_{i} n_{j}+K_{s t}\left(s_{i} s_{j}+t_{i} t_{j}\right)
$$

where $K_{n}$, and $K_{s t}$ denote asperity contact stiffness along the normal and tangential direction of the asperity contact.

At a rough interface, numerous asperity contacts of varying height overlap and orientations occur under a given loading condition. These asperity contacts can be classified into three groups: (1) those in contact but without sliding, (2) those in contact but with sliding, and (3) those not in contact. The overall interface stress can be obtained as the sum of the asperity contact forces contributed by groups (1) and (2). Utilizing the orientation distribution and height distribution introduced in section 2, we obtain the following expression for the overall interface stress, $F_{i}$, given in terms of force per unit area since $N$ is the areal asperity contact density

$$
F_{i}=N\left(\int_{r^{e}} \int_{\theta^{e}} \int_{\phi^{e}} f_{i}^{e} \xi(\theta, \phi) \sin \theta d \theta d \phi H(r) d r+\int_{r^{p}} \int_{\theta^{p}} \int_{\phi^{p}} f_{i}^{p} \xi(\theta, \phi) \sin \theta d \theta d \phi H(r) d r\right)(19
$$

In Eq. 19, the superscript $e$ denotes the domain and forces of asperity contacts that are not sliding, and the superscript $p$ denotes the domain and forces of asperity contacts 
experiencing sliding.

\section{Results}

\subsection{Sliding behavior of asperity contacts at different orientations}

Here we discuss the sliding behavior of asperity contacts subjected to a combined normal and shear displacement. We consider asperity contacts with different orientations at the same asperity contact height, $r$. We note that the asperity contact orientation are defined by $\theta$ and $\phi$ and forms a hemi-spherical surface. As we discussed before, asperity contacts with the same height $r$ experience the same global displacements $\Delta_{1}-r, \Delta_{2}$ and $\Delta_{3}$. However, the local displacements $\delta_{n}, \delta_{s}$ and $\delta_{t}$ which determine the sliding behavior of asperity contacts are different since these are functions of contact orientations. For simplicity but without loss of generality, let us consider the asperity contacts subjected to the exterior displacement that lies on the 1-3 plane of the global coordinate system and makes an oblique loading angle, $\omega$, with 1-axis as shown in Fig. 5. In this case, the asperity contacts of height, $r$, will experience a displacement of $\left(\Delta_{1}-r\right)=d$, in the direction of 1 -axis and a displacement of $\Delta_{2}=0$ and $\Delta_{3}=d \tan (\omega)$ in the directions of 2- and 3-axis, respectively, as depicted in Fig. 5. The asperity contact displacement in the local coordinate system can then be written as follows based on Eq. (7):

$\delta_{n}=d \cos (\theta)+d \tan (\omega) \sin (\theta) \sin (\phi)$

$\delta_{s}=-d \sin (\theta)+d \tan (\omega) \sin (\theta) \sin (\phi)$

$\delta_{t}=r \tan (\omega) \cos (\phi)$

Using these expressions for asperity contact displacement, we can determine whether a contact is in sliding condition. To this end, we discretize the asperity contact orientation domain into various asperity contact directions denoted by the pair $\left(\theta_{i}, \phi_{i}\right)$. For each asperity contact direction, we evaluate the local displacement using Eq. (20). When the normal displacement, $\delta_{n}\left(\theta_{i}, \phi_{i}\right)<0$, no contact exists; when the normal displacement, $\delta_{n}\left(\theta_{i}, \phi_{i}\right)>0$, and the shear displacement, $\delta_{s t}\left(\theta_{i}, \phi_{i}\right)<\mu \lambda \delta_{n}\left(\theta_{i}, \phi_{i}\right)$, 
contact exists, however is not sliding. Finally when the normal displacement, $\delta_{n}\left(\theta_{i}\right.$, $\left.\phi_{i}\right)>0$, and the shear displacement, $\delta_{s t}\left(\theta_{i}, \phi_{i}\right) \geq \mu \lambda \delta_{n}\left(\theta_{i}, \phi_{i}\right)$, contact experiences sliding. An extensive numerical search is performed using these criteria to define the asperity contact orientation groups as no-contact area, no-sliding area, or sliding area. In Figs. 6 and 7, we illustrate how the three areas evolve for different $\omega$ and $\mu \lambda$ respectively. The three areas are plotted on $\theta=0$ plane looking down along the 1-axis. The resultant polar diagram represents a projection of the asperity contact directions onto the interface $(\theta=0)$ plane. In these figures, the polar direction is $\phi$, while the radial direction is $\sin \theta$, where $\theta$ and $\phi$ are defined in Figs. 2 and 3. The points lying in the three domains then represent asperity contact directions $(\theta, \phi)$ that are experiencing no-sliding, sliding or separation. The area designated as no-sliding, given by the closed interior loop, represents the asperity contact orientations that satisfy the no-sliding criterion. The area designated as sliding, given by the space between the no-sliding area and no-contact area, represents the asperity contact orientations that satisfy the sliding criterion. The remaining directions represent asperity contacts that satisfy no-contact criterion. Under normal loading, shown in Fig. 6(a), all asperity contacts at that height $r$ are in contact, the no-sliding area is centered at $\theta=0^{\circ}$ direction and the sliding areas is given by $\theta \geq 45^{\circ}$ for the product $\mu \lambda=1$. In Fig. 6, we observe that the no-sliding area shifts towards $\phi=90^{\circ}$ and is centered at $\theta>0^{\circ}$ as the interface is subjected to shear along the 3 -axis. We find that as we increase the loading angle, $\omega$, the no-sliding domain moves closer to the horizon accompanied by an expanding no-contact area. We also find that the extent of sliding area depends upon the contact parameter given by the product, $\mu \lambda$., as shown in Fig. 7.

\subsection{Stress-Displacement Behavior under Combined Normal-Shear Loading}

To investigate the stress-displacement behavior of rough interface under combined normal-shear loading for both isotropic and anisotropic interface, we design the following loading procedure. As illustrated in Fig. 8, we first apply a normal displacement, $\Delta_{1}$, subsequently, we apply a shear displacement in the 2-direction, $\Delta_{2}$, 
followed by shear displacement in the 3-direction, $\Delta_{3}$. For this loading sequence, we numerically evaluate the discretized Eq. (19) as described in the previous section. The interface properties used for our example computations are tabulated in Table 1.

Fig. 9 shows the resultant stress-displacement curves. During loading step 1, stress, $F_{1}$, increases as interface undergoes closure. Under shear loading in step 2, the normal stress, $F_{l}$, increases although the normal displacement, $\Delta_{l}$, is kept constant. The increase in normal stress shows the coupling between the normal and shear behavior induced by the shear loading and is indicative of the fact that the interface is constrained from dilating. As we now apply the shear loading in step 3 while keeping the normal displacement, $\Delta_{1}$, and the shear displacement, $\Delta_{2}$, we see further coupling develop between the normal-shear and shear directions. During this loading step, we find a significant difference between the shear stresses, $F_{1}$ and $F_{2}$, although the corresponding shear displacements may be the same, showing a clear manifestation of the loading induced anisotropy. At the asperity contact-level, the no-contact, no-sliding and sliding areas evolve in response to the applied load-path. The result, of course, is a highly load path dependent overall behavior. To further illustrate how the stress-displacement behavior changes as a function of the surface characteristics, we plot the stress-displacement curves for asperity contact friction coefficient, $\mu=0.3$, in Fig. 9, for two different asperity contact orientation distributions in Fig. 10, for two different asperity contact height distributions in Fig. 11, and for anisotropic asperity contact distributions in Fig. 12. As expected, the overall shear behavior is softer for the lower asperity contact friction coefficient; however, the normal behavior becomes more dilatant as seen from a crossover of the normal stress, $F_{1}$, as the shear displacement is increased.

In Fig. 10, the stress-displacement curves are compared for asperity contact orientation distribution parameter $a=1.5$ and 3 . We observe that the normal behavior of the two interfaces is similar while the shear behavior shows significant differences. Since the meridional extent of asperity contact orientation for $a=1.5$ is much greater 
than that for $a=3.0$, a significantly stiffer shear behavior is obtained. We also not that the interface with $a=3.0$ shows shear softening in the 2-direction as the interface is sheared in the 3-direction. In Fig. 11, the stress-displacement curves obtained for rough interface given by the asperity contact height distribution parameters $\alpha=6.14$, $\beta=3.52 \mu \mathrm{m}$ are compared to those obtained for a smooth interface whose asperity contact height distribution parameters are taken as $\alpha=3.82, \beta=1.15 \mu m$. Clearly, surface roughness plays an important role in stress-displacement behavior. As seen in Fig. 11, the smoother interface shows a stiffer behavior. Further, in Fig. 12, we compare the behavior for isotropic and anisotropic interfaces. The anisotropy parameters and the initial contact distributions are given in the right plot of Fig. 3 $(a=1, b=-1, c=1 / 3)$. We note that that anisotropy has only a small affect on the normal behavior. However, in contrast to the isotropic interface, the stresses for the anisotropic interface in the 2- and 3-directions are different even though the applied displacements in the two directions are the same.

\section{Concluding Remarks}

In this study, we have utilized a micromechanical method, in which the surface roughness is modeled using a statistical approach utilizing asperity contact height and orientation distributions. We incorporate Hertz-Mindlin fundamental solutions and sliding at the asperity contact in our analysis. The micromechanical method is found to be applicable for modeling both the inherent anisotropy of the surface as well as the anisotropy induced by shear loading. In particular, we have investigated how the stress-displacement relationship evolves as the interface is subjected to shear loading whose direction is varied. We have obtained the boundaries between elastic, sliding and non-contacting asperity contact orientations as a function of the loading condition and the asperity Poisson's ratio and friction coefficient. We show for the first time that an initially isotropic interface can exhibit highly anisotropic behavior when subjected to a shear preload. Moreover, the normal and shear behavior are coupled in the presence of shear loading. We also found that coupling effect between normal 
and shear direction is much stronger in rough interface than that in smooth interface. 


\section{References}

[1] Hertz H, On the contact of elastic solids. Journal fur de reine und angewandte Mathematik 1881; 92: 156-71.

[2] Hertz $\mathrm{H}$, On the contact of rigid elastic solids and on hardness. Verhandlungen des Vereins zur Befoderung des Gewerbefleisses 1882.

[3] Johnson KL, Contact mechanics. Cambridge: Cambridge University Press; 1985.

[4] Wriggers P, Computational contact mechanics. 2nd ed. Berlin ; New York: Springer; 2006.

[5] Wriggers P, Laursen TA, Computational Contact Mechanics. Vienna: CISM; 2008.

[6] Shankar S, Mayuram MM, A finite element based study on the elastic-plastic transition behavior in a hemisphere in contact with a rigid flat. Journal of Tribology-Transactions of the Asme 2008; 130(4): 044502-1-6.

[7] Hyun S, Pei L, Molinari JF, Robbins MO, Finite-element analysis of contact between elastic self-affine surfaces. Physical Review E 2004; 70(2): 026117-1-12.

[8] Laursen TA, Computational Contact and Impact Mechanics. New York: Springer; 2002.

[9] Yoshioka N, Elastic Behavior of Contacting Surfaces under Normal Loads - a Computer-Simulation Using 3-Dimensional Surface Topographies. Journal of Geophysical Research-Solid Earth 1994; 99(B8): 15549-60.

[10] Majumdar A, Bhushan B, Role of Fractal Geometry in Roughness Characterization and Contact Mechanics of Surfaces. Journal of Tribology-Transactions of the Asme 1990; 112(2): 205-16.

[11] Persson BNJ, Albohr O, Tartaglino U, Volokitin AI, Tosatti E, On the nature of surface roughness with application to contact mechanics, sealing, rubber friction and adhesion. Journal of Physics-Condensed Matter 2005; 17(1): R1-R62.

[12] Yang J, Komvopoulos K, A mechanics approach to static friction of elastic-plastic fractal surfaces. Journal of Tribology-Transactions of the Asme 2005; 127(2): 315-24.

[13] Ciavarella M, Delfine V, Demelio V, A new 2D asperity model with interaction for studying the contact of multiscale rough random profiles. Wear 2006; 261(5-6): 556-67.

[14] Buczkowski R, Kleiber M, Statistical Models of Rough Surfaces for Finite Element 3D-Contact Analysis. Archives of Computational Methods in Engineering 2009; 16(4): 399-424.

[15] Greenwood JA, Williams JB, Contact of Nominally Flat Surfaces. Proceedings of the Royal Society of London Series a-Mathematical and Physical Sciences 1966; 295(1442): 300-19.

[16] Whitehouse DJ, Archard JF, Properties of Random Surfaces of Significance in Their Contact. Proceedings of the Royal Society of London Series a-Mathematical and Physical Sciences 1970; 316(1524): 97-121. 
[17] Greenwood JA, Tripp JH, The contact of two nominally flat rough surfaces. Proceedings of the Institution of Mechanical Engineers 1971; 185: 625-33.

[18] Nayak PR, Random Process Model of Rough Surfaces. Journal of Lubrication Technology 1971; 93(3): 398-407.

[19] Bush AW, Gibson RD, Thomas TR, Elastic Contact of a Rough Surface. Wear 1975; 35(1): 87-111.

[20] Bush AW, Gibson RD, Keogh GP, Strongly Anisotropic Rough Surfaces. Journal of Lubrication Technology-Transactions of the Asme 1979; 101(1): 15-20.

[21] Adler RJ, Firman D, A Non-Gaussian Model for Random Surfaces. Philosophical Transactions of the Royal Society of London Series a-Mathematical Physical and Engineering Sciences 1981; 303(1479): 433-62.

[22] Mccool JI, S.S.Gassel, The contact of two surfaces having anisotropic roughness geometry, in Energy technology, Special Publication. 1981, American Society of Lubrication Engineers: New York. 29-38.

[23] Brown SR, Scholz CH, Closure of Random Elastic Surfaces in Contact. Journal of Geophysical Research-Solid Earth and Planets 1985; 90(Nb7): 5531-45.

[24] Brown SR, Scholz CH, Closure of Rock Joints. Journal of Geophysical Research-Solid Earth and Planets 1986; 91(B5): 4939-48.

[25] Chang WR, Etsion I, Bogy DB, An Elastic-Plastic Model for the Contact of Rough Surfaces. Journal of Tribology-Transactions of the Asme 1987; 109(2): 257-63.

[26] Yoshioka N, Scholz CH, Elastic Properties of Contacting Surfaces under Normal and Shear Loads .1. Theory. Journal of Geophysical Research-Solid Earth and Planets 1989; 94(B12): 17681-90.

[27] Yoshioka N, Scholz CH, Elastic Properties of Contacting Surfaces under Normal and Shear Loads .2. Comparison of Theory with Experiment. Journal of Geophysical Research-Solid Earth and Planets 1989; 94(B12): 17691-700.

[28] Misra A, Mechanistic model for contact between rough surfaces. Journal of Engineering Mechanics-Asce 1997; 123(5): 475-84.

[29] Archard JF, Elastic Deformation and the Laws of Friction. Proceedings of the Royal Society of London Series a-Mathematical and Physical Sciences 1957; 243(1233): 190-205.

[30] Cooper MG, Mikic BB, Yovanovich MM, Thermal Contact Conductance. International Journal of Heat and Mass Transfer 1969; 12(3): 279-300.

[31] Tabor D, Friction - the Present State of Our Understanding. Journal of Lubrication Technology-Transactions of the Asme 1981; 103(2): 169-79.

[32] Zavarise G, Borri-Brunetto M, Paggi M, On the reliability of microscopical contact models. Wear 2004; 257(3-4): 229-45.

[33] Zavarise G, Borri-Brunetto M, Paggi M, On the resolution dependence of micromechanical contact models. Wear 2007; 262(1-2): 42-54.

[34] Nayak PR, Random Process Model of Rough Surfaces in Plastic Contact. Wear 1973; 26(3): 305-33. 
[35] Misra A, Micromechanical model for anisotropic rock joints. Journal of Geophysical Research-Solid Earth 1999; 104(B10): 23175-87.

[36] Misra A, Huang S, Micromechanics based stress-displacement relationships of rough contacts: Numerical implementation under combined normal and shear loading. Cmes-Computer Modeling in Engineering \& Sciences 2009; 52(2): 197-215.

[37] Mindlin RD, Deresiewicz H, Elastic Spheres in Contact under Varying Oblique Forces. Journal of Applied Mechanics-Transactions of the Asme 1953; 20(3): 327-44. 


\section{List of nomenclature}

$a, b$ and $c=$ orientation distribution parameters

$d=$ asperity contact displacement in normal direction

$e=$ non-sliding asperity contact

$f_{i}=$ asperity contact forces

$F_{i}=$ overall interface stress

$G=$ shear modulus

$H=$ asperity contact height distribution

$K_{i j}=$ asperity contact stiffnesses

$K_{n}, K_{s t}=$ asperity contact stiffness along the normal and the tangential directions

$n, s$ and $t=$ spherical coordinate direction vector

$N=$ asperity contact density given as number of asperity contact per unit area

$p=$ sliding asperity contact

$r=$ composite topography height or asperity contact height

$R=$ radius of asperity

$\alpha, \beta=$ height distribution parameters

$\delta_{j}=$ asperity contact displacement

$\Delta_{i}=$ interface displacement

$\phi$ and $\theta=$ spherical coordinates

$\eta=$ plasticity parameter

$\lambda=$ material constant

$\mu=$ asperity contact friction

$v=$ Poisson's ratio

$\omega=$ angle between the asperity contact normal and the 1-axis in the 1-3 plane

$\xi=$ density function of asperity contact orientations, 


\section{List of Figures}

Figure 1 Distribution of asperity contact heights

Figure 1 Schematic depiction of the micromechanical modeling methodology for rough interfaces.

Figure 3: Asperity contact orientation distribution, $\xi(\theta, \phi)$ : left frame is for isotropic interface $(a=1, b=1 / 3, c=0)$ and right frame is for anisotropic interface $(a=1, b=-1, c=1 / 3)$. Grayscales indicate the probability density of finding asperity contact in a given orientation defined by the coordinate system given in the inset. The plots are in spherical coordinates where the radius is $\xi(\theta, \phi)$.

Figure 4 Asperity contact displacement, $d$, at height $r$ for a normal interface displacement of $\Delta_{l}$.

Figure 5 Oblique loading angle $\omega$.

Figure $6 \omega$ effect on sliding and no sliding distribution $(\mu \lambda=1)$.

Figure $7 \mu \lambda$ effect on sliding and no sliding distribution $(\omega=\pi / 4)$.

Figure 8 Loading procedure.

Figure 9 Effect of friction coefficient on normal and shear behavior.

Figure 10 Effect of asperity orientation distribution parameter, $a$, on the normal and shear behavior.

Figure 11 Effect of roughness on normal and shear behavior.

Figure 12 Effect of anisotropy on normal and shear behavior.

\section{List of Tables}

Table 1 Interface property. 
Table 1 Interface property.

asperity density friction coefficient plasticity parameter orientation distribution

$N=1000 / \mathrm{mm}^{2} \quad \mu=1 \quad \eta=1 / 2$

$a=3, b=0, c=0$

Radius shear modulus Poisson's ratio height distribution

$R=200 \mu m \quad G=80(\mathrm{GPa}) \quad v=0.3 \quad \alpha=5.12, \beta=2.35 \mu \mathrm{m}$ 


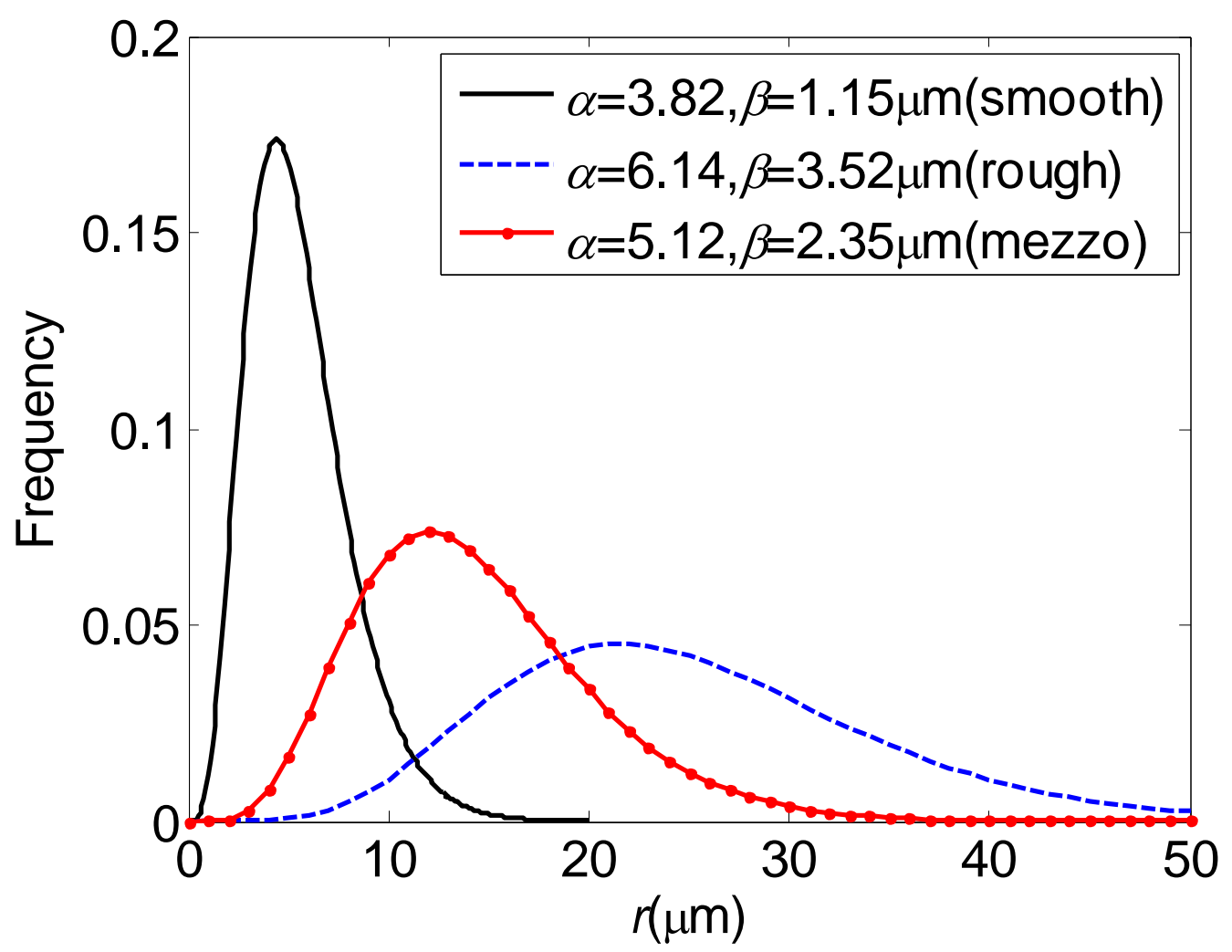

Figure 1 Distribution of asperity contact heights 


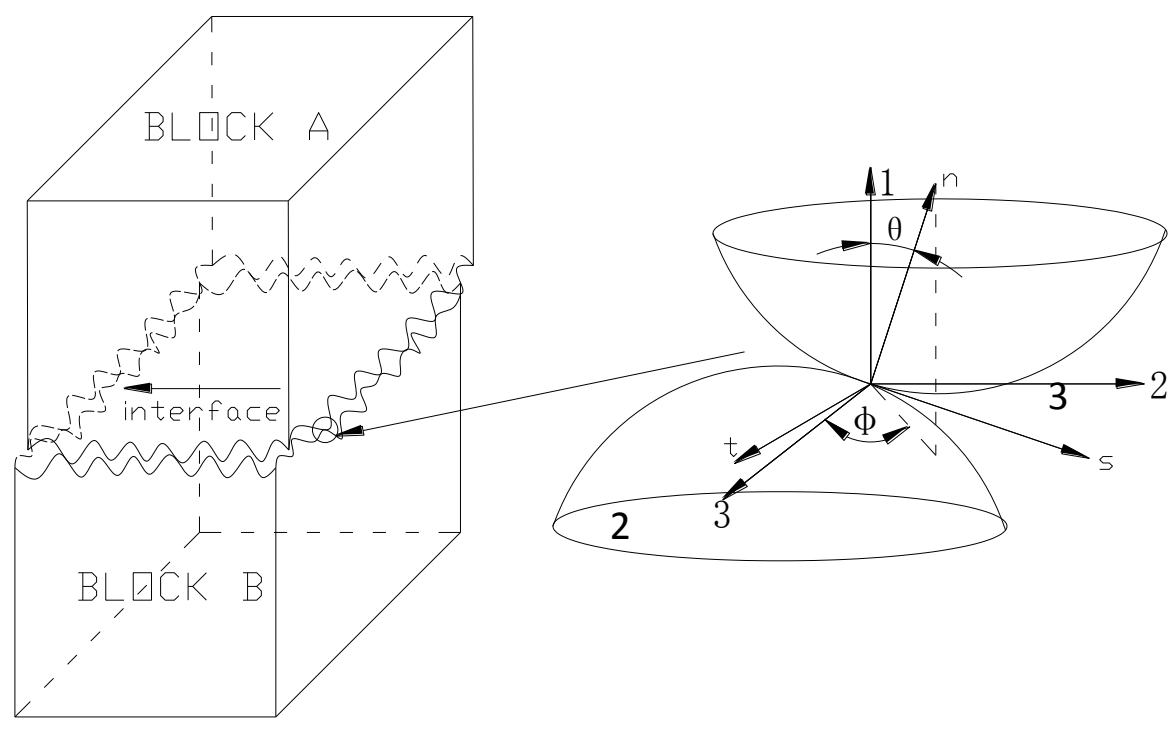

Figure 2 Schematic depiction of the micromechanical modeling methodology for rough interfaces 

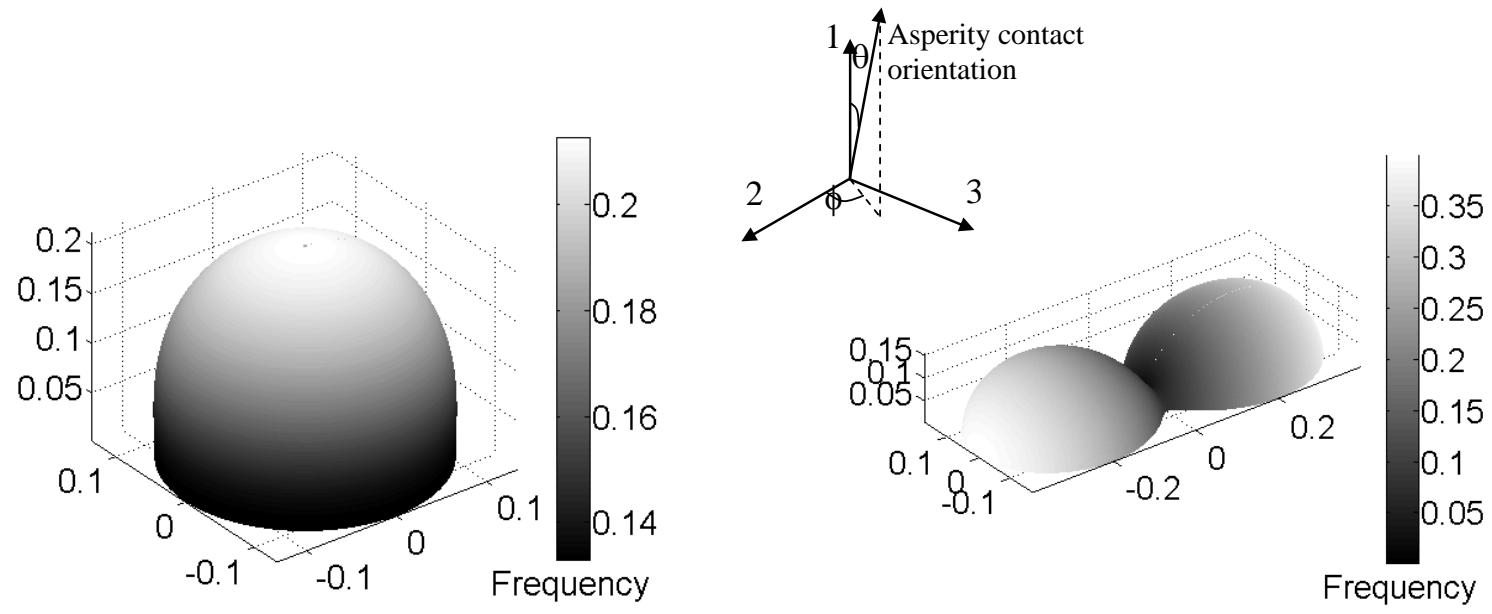

Figure 3: Asperity contact orientation distribution, $\xi(\theta, \phi)$ : left frame is for isotropic interface $(a=1, b=1 / 3, c=0)$ and right frame is for anisotropic interface $(a=1, b=-1, c=1 / 3)$. Grayscales indicate the probability density of finding asperity contact in a given orientation defined by the coordinate system given in the inset. The plots are in spherical coordinates where the radius is $\xi(\theta, \phi)$. 


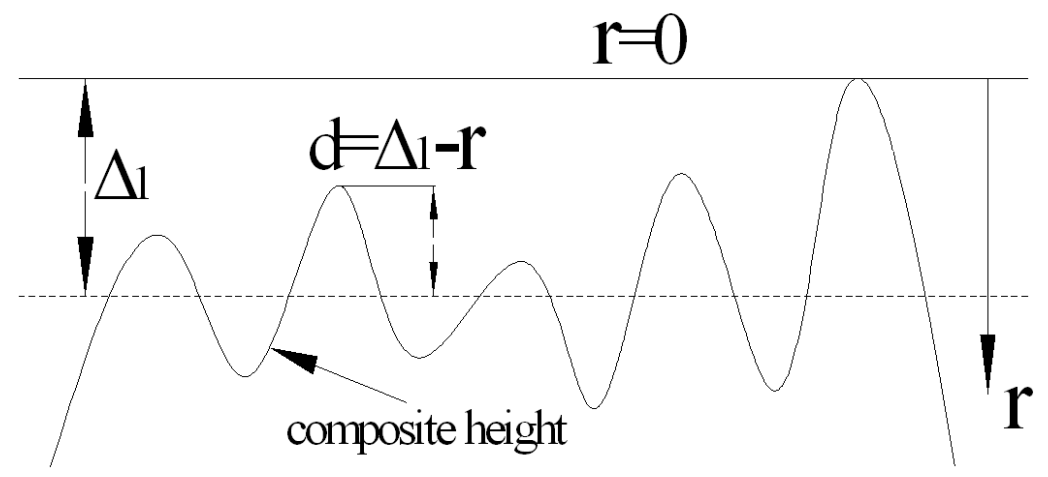

Figure 4 Asperity contact displacement, $d$, at height $r$ for a normal interface displacement of $\Delta_{l}$. 


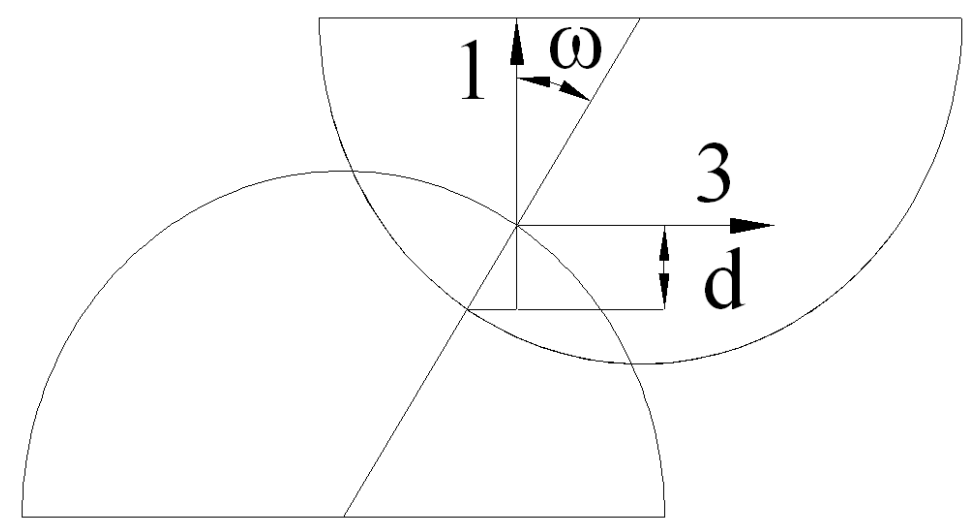

Figure 5 Oblique loading angle $\omega$. 


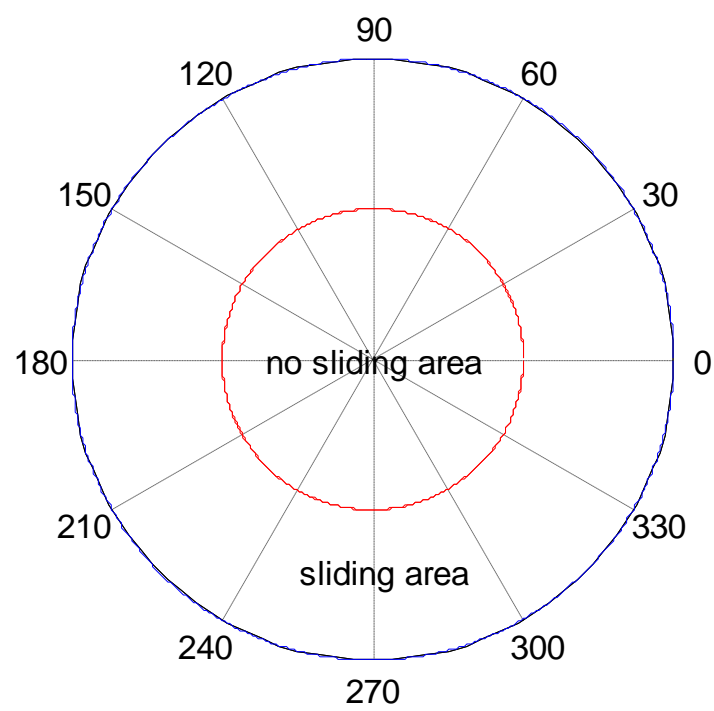

(a) $\quad \omega=0$

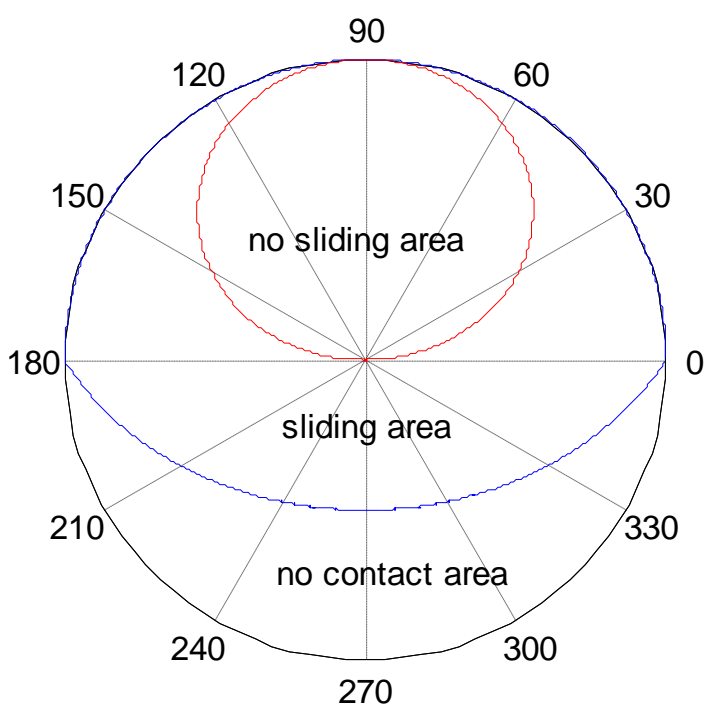

(c) $\omega=\pi / 4$

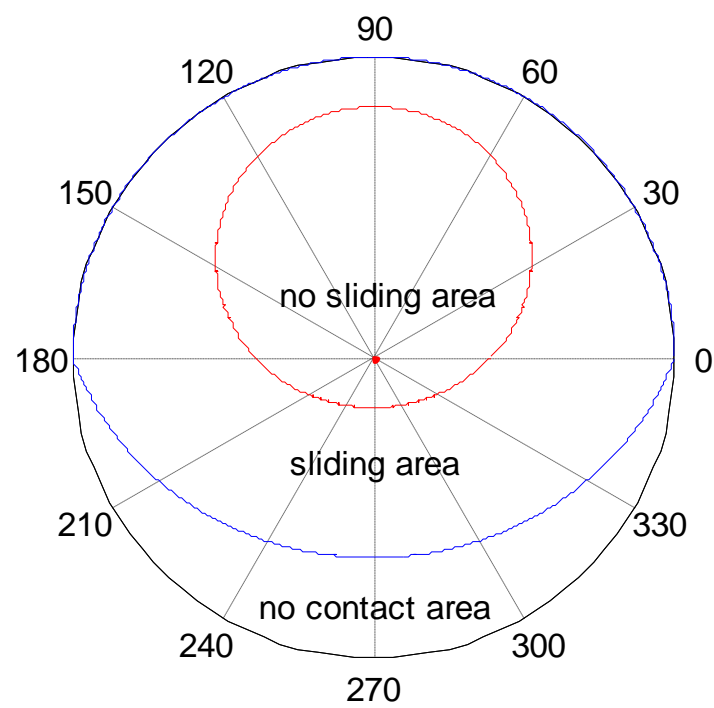

(b) $\quad \omega=\pi / 6$

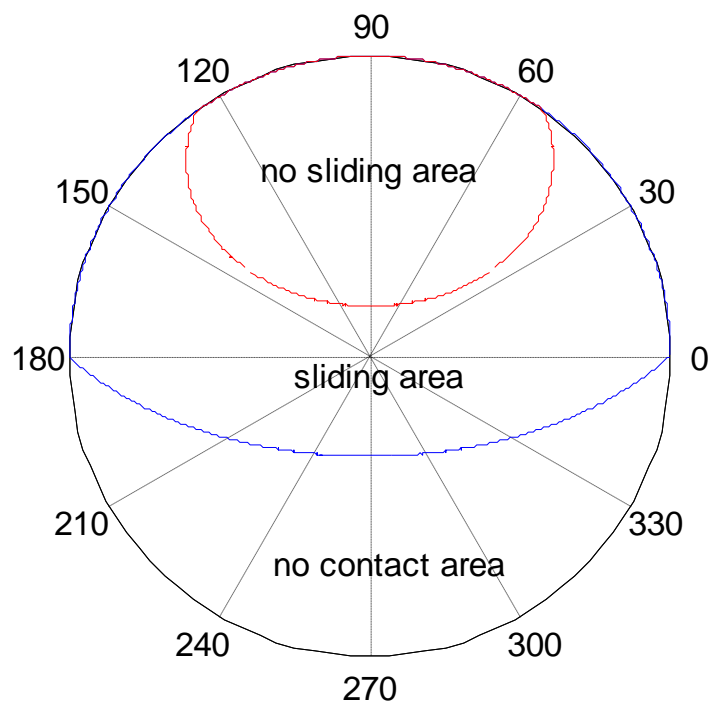

(d) $\omega=\pi / 3$

Figure $6 \omega$ effect on sliding and no sliding distribution $(\mu \lambda=1)$ 


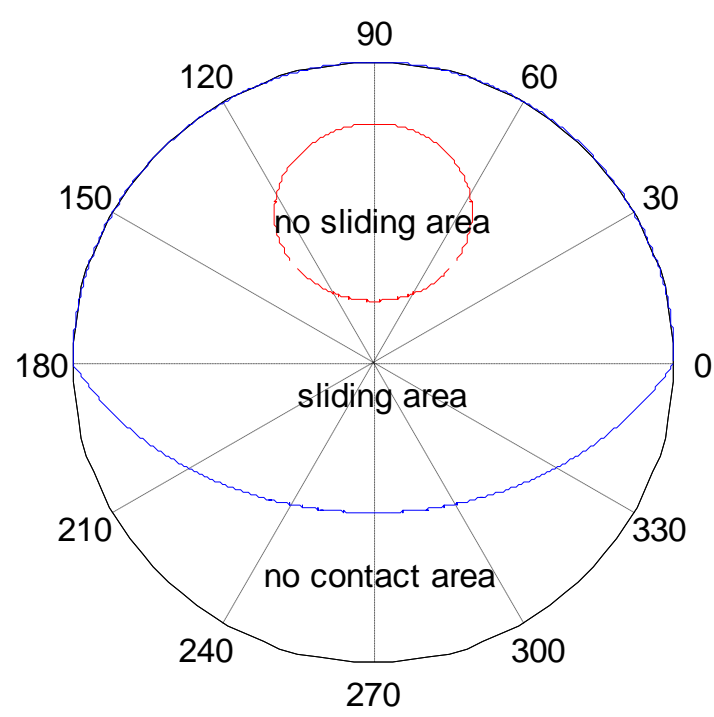

(a) $\mu \lambda=0.5$

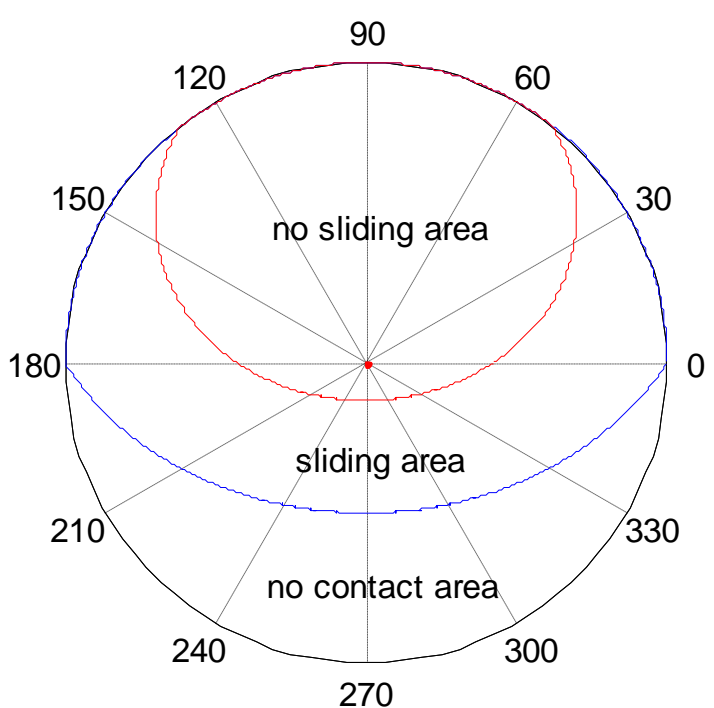

(b) $\mu \lambda=1.5$

Figure $7 \quad \mu \lambda$ effect on sliding and no sliding distribution $(\omega=\pi / 4)$ 


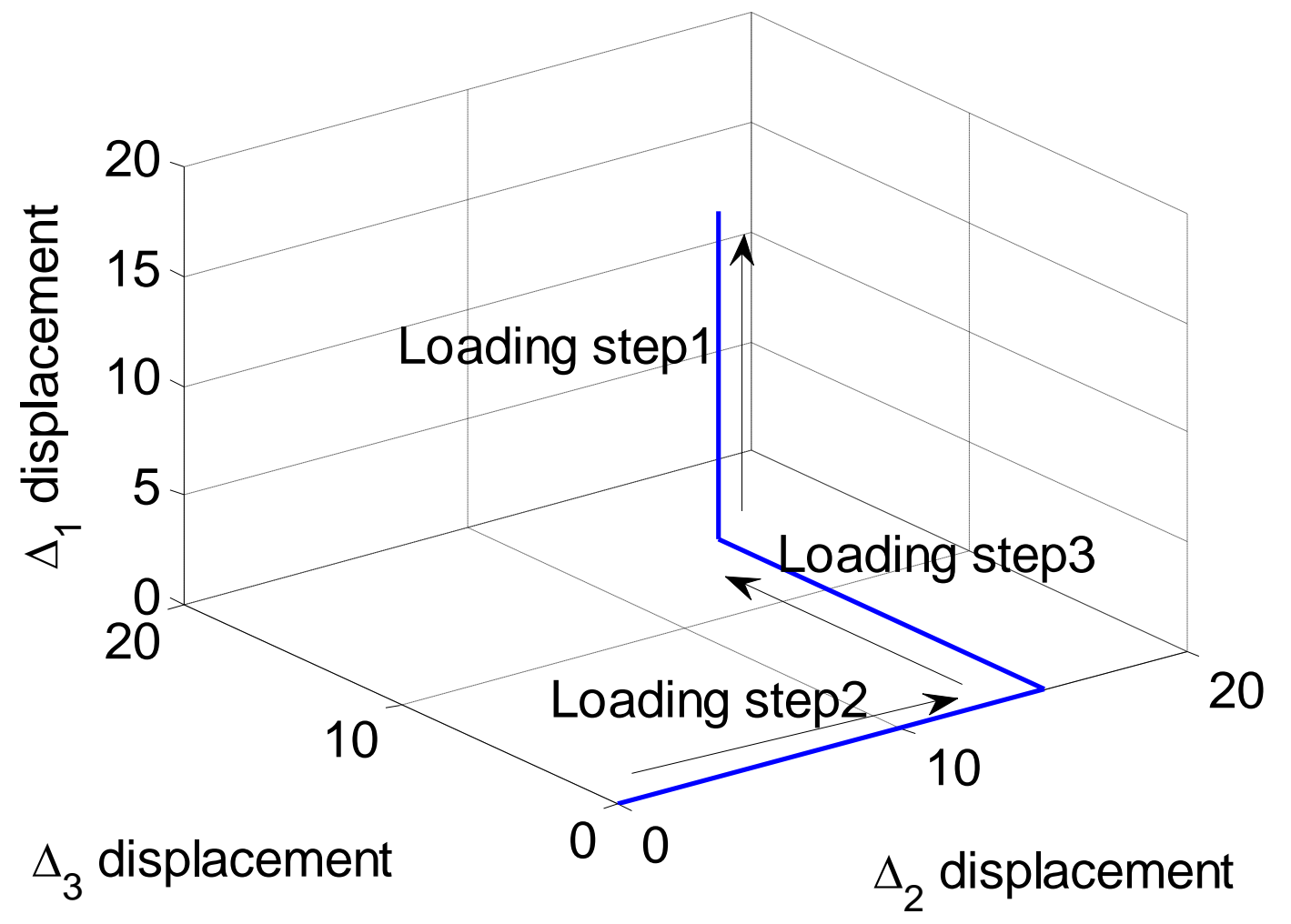

Figure 8 Loading procedure 


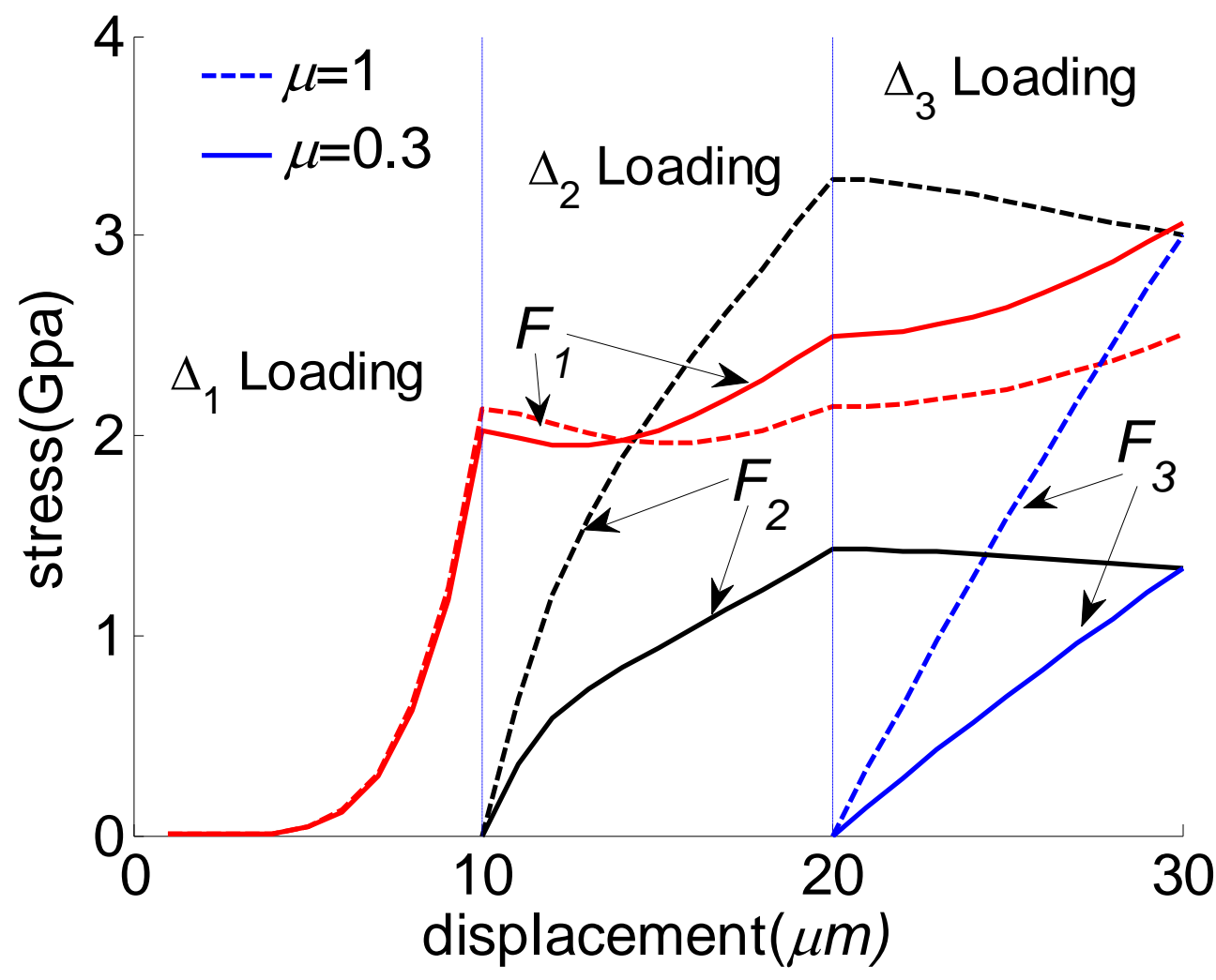

Figure 9 Effect of friction coefficient on normal and shear behavior. 


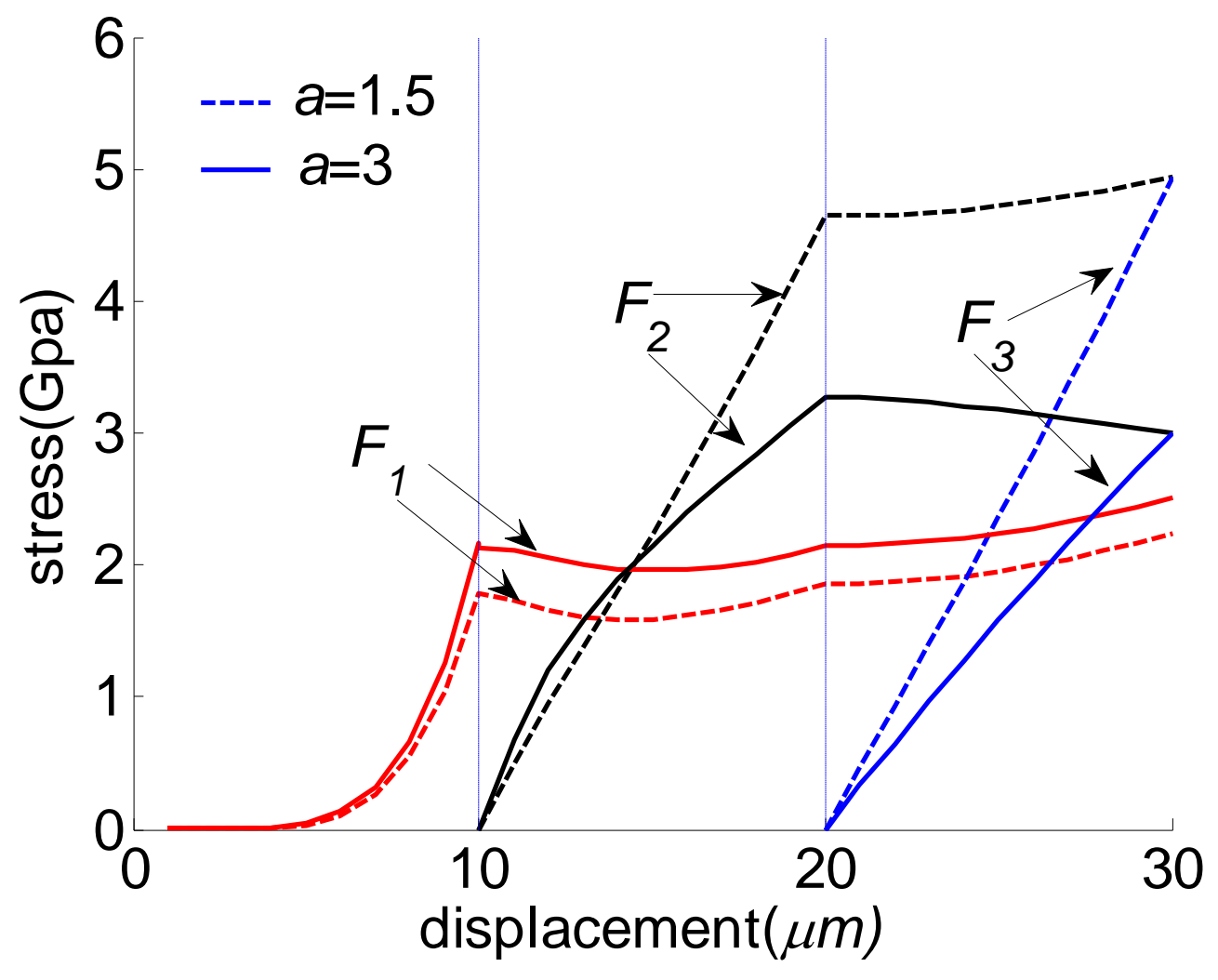

Figure 10 Effect of asperity orientation distribution parameter, $a$, on the normal and shear behavior. 


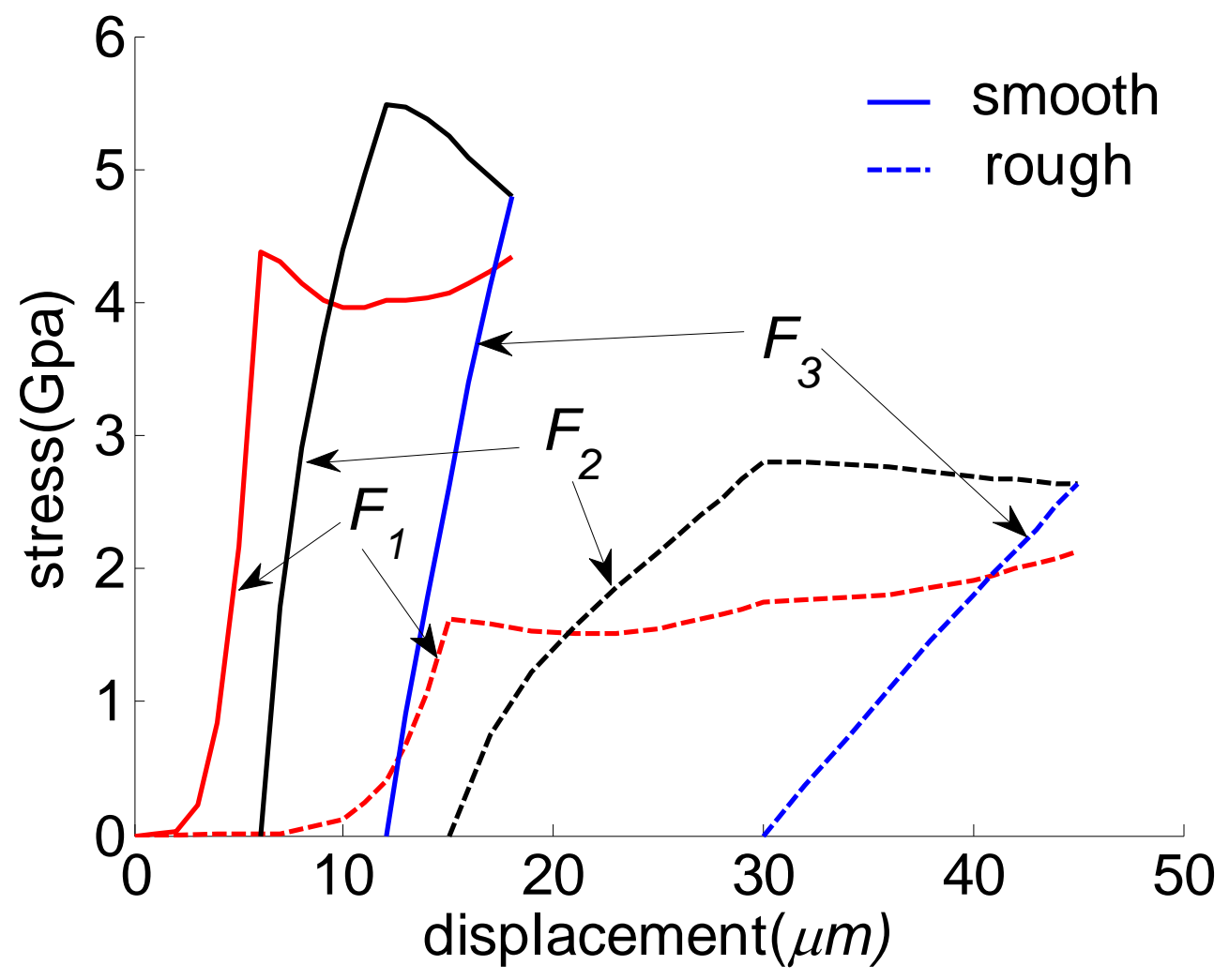

Figure 11 Effect of roughness on normal and shear behavior. 


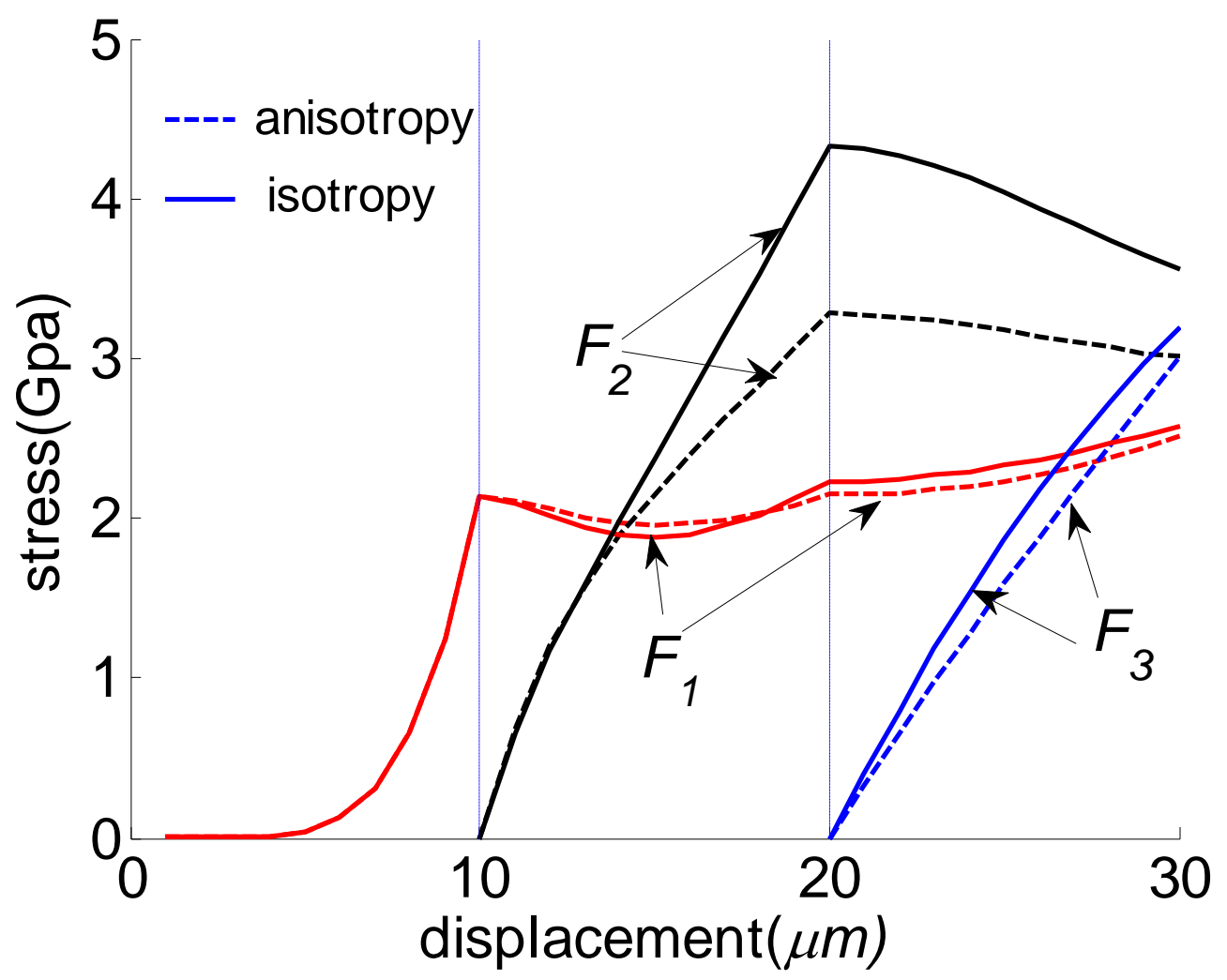

Figure 12 Effect of anisotropy on normal and shear behavior. 